\title{
Sous le charme de la méchante fleur
}

Le «minimal art» est la manière de donner à l'art un sens des plus éloquents avec les moyens les plus simples. Alors que les travaux d'Anna, qui enrichissent le BMS depuis toujours, ne font certes pas partie de cette catégorie du point de vue de l'histoire de l'art, ils répondent certainement à cette exigence de simplicité. Un regard vers la page de droite saura vous en convaincre.

Certes, on peut exagérer en tout. Et je l'avoue, je n'ai pas pu empêcher cette impression de surgir à la première lecture du livre d'Anna récemment paru*. Le premier jugement en la matière ne correspondant pas à l'opinion finale, cet aveu peut se faire sans problème. Sinon, le rédacteur soussigné garderait prudemment pour lui son impression de l'œuvre ou déléguerait à d'autres, de manière plus ou moins élégante, le soin d'en rendre compte.

Mais reprenons les choses par le début. «Böse Blume», tel est le titre du livre. Un titre merveilleusement poétique, ouvrant un vaste champ

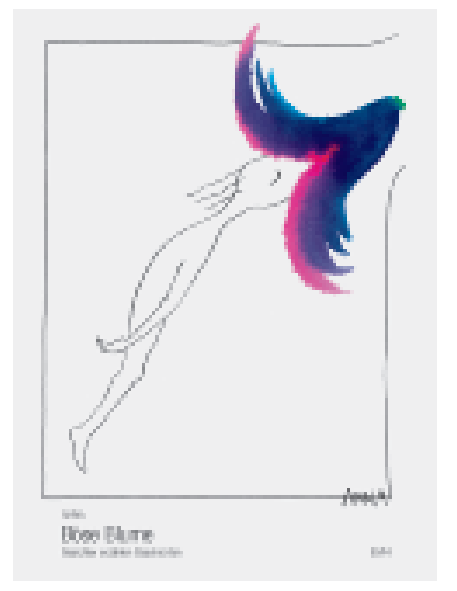

d'associations mais définissant aussi avec à propos et précision l'objet choisi, tout en restant dans un certain flou métaphorique. L'ouvrage traite de drogues et de dépendances, mais surtout de personnages et d'histoires qui ont un rapport à la toxicomanie, comme le relève l'auteur ellemême dans son introduction. La fleur est dotée d'une attirance magique mais sa beauté et son parfum sont dangereux pour qui s'en approche trop. Le papaver somniferum, le pavot somnifère, peut être désigné comme l'archétype de la fleur nuisible. Son suc blanchâtre donne l'opium, symbole de la drogue en soi.

L'image de titre nous la donne, cette fleur méchante largement ouverte, dont la corolle miroitante, dans les tons foncés violets, bleus et verts, renferme un personnage éthéré qui y plonge la tête. Sa position légère fait songer à un sauteur de ski en vol, impression renforcée par les cheveux au vent. Le vol d'un rêveur échappé de la lourdeur terrestre.

Il est impressionnant de voir comment Anna sait pousser à l'extrême le principe cité en introduction de l'art minimal. On ne peut faire plus dépouillé. Bien que la partie de la bouche soit cachée par les pétales, on sait que l'être planant sourit aux anges: la position du corps et surtout le trait des paupières fermées en sont presque certainement la preuve. Ce mode de travail réductionniste est typique aussi des 40 portraits qui constituent l'ouvrage. L'auteur donne à l'image une force et une clarté que l'on pourrait caractériser d'«essentielles». Cette force et cette clarté peuvent toutefois devenir difficiles à supporter. Peut-être cela tient-il au fait que certains de ces portraits ont été retravaillés à l'aide d'une photocopieuse par leurs sujets, jusqu'à ce que leur visage corresponde à l'image faussée qu'ils avaient d'eux-mêmes? On ne tombe, soit dit en passant, sur aucun sourire radieux.

Anna croit dans la force de ses dessins et livre au spectateur un minimum de texte d'accompagnement. Cela peut apparaître au premier abord comme une faiblesse de l'ouvrage. On se sent un peu seul et désemparé. Mais si on relève le défi et que l'on se penche sur ces visages, le concept est très convaincant. Des commentaires plus complets et des explications détaillées sur les biographies et l'anamnèse des toxicomanies pourraient faciliter l'approche, mais détourner de l'essentiel tout en favorisant un regard plus superficiel et voyeuriste. Ce serait pour ce thème-là le pire des «retournements», pour conclure sur une formule de Dürrenmatt.

Bruno Kesseli suisses SA; 2006

ISBN-13: 978-3-7965-2289-5. 